\title{
La universidad pública en la transferencia tecnológica: ¿losa de 20 toneladas?
}

\author{
Armando Miranda Zea, Omar García Ponce \\ de León \& Ricardo Pérez Mora
}

Resumen:

El artículo tiene como objetivo comprender cómo el clima organizacional de las Universidades Públicas Estatales del país influye en la construcción de las trayectorias académico-profesionales de los profesores-investigadores que deciden participar en procesos de transferencia tecnológica. Durante la investigación se utilizó la metodología cualitativa. Se aplicaron entrevistas a profundidad para reconstruir el mundo de significados de los sujetos de estudio y rescatar sus experiencias en torno a la transferencia de conocimiento. En el estudio se encontró que las UPE's poseen rutinas burocráticas, creencias, valores, financiamientos, personal e infraestructura que pueden significar un obstáculo para las expectativas de los profesores-investigadores innovadores.

Palabras clave:

ambiente laboral; profesor de universidad; universidad pública; vida profesional; transferencia de tecnología. 


\section{A universidade pública em transferência tecnológica: laje de 20 toneladas?}

Resumo: O objetivo do artigo é compreender como o clima organizacional das Universidades Públicas Estaduais do país influencia a construção das trajetórias acadêmico-profissionais dos professores-pesquisadores que decidem participar de processos de transferência de tecnologia. Durante a pesquisa, utilizou-se metodologia qualitativa. Entrevistas em profundidade foram aplicadas para reconstruir o mundo dos significados dos sujeitos do estudo e recuperar suas experiências em relação à transferência de conhecimento. No estudo verificou-se que as UPEs possuem rotinas, crenças, valores, financiamentos, pessoal e infraestrutura burocráticos que podem significar um obstáculo às expectativas dos inovadores professores-pesquisadores.

Palavras-chave: ambiente de trabalho; professor de universidade; universidade pública; vida profissional; transferência de tecnologia.

\section{The public university in technological transfer: 20-tone slab?}

Abstract: The article aims to understand how the organizational climate of state public universities in the country influences the construction of academic-professional teachers-researchers who choose to participate in technology transfer processes trajectories. Qualitative methodology was used during the investigation. Depth interviews were applied to reconstruct the world of meaning of the study subjects and redeem their experiences about knowledge transfer. The study found that UPE's own bureaucratic routines, beliefs, values, financing, personnel and infrastructure that can mean an obstacle to the expectations of teachers and researchers innovators.

Keywords: work environment; University professor; public university; professional life; technology transfer.

\section{L'université publique du transfert technologique: dalle 20 tons?}

Résumé: L'objectif de l'article est de comprendre comment le climat organisationnel de l'État public des universités du pays influence la construction des trajectoires universitaires-professionnelles des professeurs-chercheurs qui décident de participer à des processus de transfert de technologie. Au cours de la recherche, une méthodologie qualitative a été utilisée. Des entretiens approfondis ont été appliqués pour reconstruire le monde des significations des sujets d'étude et pour récupérer leurs expériences en matière de transfert de connaissances. Dans l'étude, i a été constaté que I'UPE avait des routines bureaucratiques, des croyances, des valeurs, des financements, du personnel et des infrastructures pouvant constituer un obstacle aux attentes des enseignants-chercheurs innovants.

Mots clés: ambiance de travail; professeur d'université; université publique; vie professionnelle; transfert de technologie. 


\section{Introducción}

El objetivo del artículo es comprender la manera en que el clima organizacional de las Universidades Públicas Estatales del país influye en la construcción de las trayectorias académico-profesionales de los profesores-investigadores que participan en procesos de transferencia tecnológica. Las trayectorias académico-profesionales reflejan las etapas que el sujeto que se dedica a la docencia y la investigación vive a lo largo de su carrera profesional, las cuales reflejan las actividades que desarrolla y los espacios donde las construye (Jiménez, 2009). El clima organizacional es la manera en que los elementos objetivos de una organización influyen en la percepción, el comportamiento y las decisiones de sus miembros (Bermúdez, Pedraza y Rincón, 2015). Los elementos objetivos son su estructura, conformada por reglas y rutinas, y su cultura, compuesta por valores, creencias, rituales y símbolos.

La universidad está siendo cuestionada acerca de su papel en la sociedad porque se quiere que repercuta más allá de sus labores de profesionalización de recursos humanos e investigación científica. Se le exige que tenga como prioridad fungir como actor protagónico en el desarrollo de la economía, a través de la movilización del conocimiento científico, la vinculación y la innovación (Galindo, Sanz y De Benito, 2011). Así nace la Tercera Misión de la Universidad, la cual tiene el objetivo de difundir e implementar el conocimiento fuera del ámbito académico para afectar a las instancias receptoras mediante la innovación tecnológica y social (Clark, 1998; Gibbons et al., 1997). Los saberes de los científicos no solo son compartidos en medios académicos convencionales, sino que son exportados a contextos en los que se requiere su aplicación.

La transferencia de conocimiento inicia cuando las organizaciones y las comunidades requieren de los servicios de los investigadores universitarios para resolver problemas (Lee y Win, 2004). Es en este momento cuando los científicos comienzan a trabajar en ideas nuevas para desarrollar conocimiento útil para sus clientes. En ese instante la universidad productora y la organización receptora firman un convenio legal para establecer "las reglas del juego", mantener una relación cordial y recíproca y evitar la fuga de información confidencial de las ideas de los científicos. Esta tarea se facilita cuando las universidades tienen una actitud proactiva hacia la innovación y se convierten en Universidades Emprendedoras.

Las Universidades Emprendedoras contribuyen al desarrollo socioeconómico de sus regiones, mediante la cultura de "emprendurismo universitario" y una actitud de competitividad y liderazgo (de Arteche, Santucci y Welsh, 2013; Guerrero y Urbano, 2012). Las universidades que desarrollan proyectos empresariales son organizaciones que adoptan valores de las empresas privadas para comercializar el conocimiento, aumentar la competitividad de sus socios y obtener sus propios recursos. Etzkowitz (2003) sostiene que la universidad emprendedora tiene que ser una incubadora de ideas novedosas. Para ello, la universidad necesita combinar una serie de factores. 
Para Clark (1998), una universidad que aspire a ser emprendedora necesita una estructura organizacional flexible con Oficinas de Transferencia Tecnológicas eficaces, una directiva capaz de gestionar el conocimiento, personal académico motivado a emprender proyectos tecnológicos, fuentes internas y externas de financiamiento y una cultura de emprendimiento consolidada. Pero ¿qué sucede cuando las universidades públicas están lejos de ser instituciones que consolidan la transferencia de conocimiento por poseer un clima organizacional anquilosado? Los entrevistados relatan sus experiencias y describen los factores que son un obstáculo institucional para su labor de emprendimiento.

\section{Metodología}

La metodología que se empleó es cualitativa porque se quiere comprender el fenómeno estudiado, a partir de los testimonios orales y las interpretaciones de los sujetos de estudio. De acuerdo con Hernández, Fernández y Baptista (2006) y Rodríguez, Gil y García (1996), la investigación cualitativa nos permite comprender y reinterpretar mediante la empatía el mundo de significados, percepciones, motivaciones e interpretaciones subjetivas de los individuos que le dan sentido y coherencia a su acción social dentro de un ambiente determinado. Lo que se quiere es reinterpretar el mundo simbólico de los investigadores tal y como lo experimentan en un ambiente natural y cotidiano de sentido común (Denzin y Lincoln, 2000).

El método que se manejó es fenomenológico porque se centra en la comprensión más que en la explicación de los significados que construyen el entorno social de los sujetos (Rizo, 2006). Los métodos cualitativos tienden a ser “... relativistas, holistas, descriptivos/exploratorios, subjetivos, inductivos, especulativos/ilustrativos, ideográficos, interpretativistas, orientados a exponer el significado para los actores, etc. ..." (Cortés, 2000, p.82). Esto quiere decir que son relativistas porque se comprende la realidad desde la perspectiva subjetivad el actor sin buscar la verdad. También son holistas porque se interpreta un fenómeno desde todas sus aristas. De igual manera son descriptivos e inductivos porque se hace una descripción de las causas del comportamiento de los sujetos a partir de los datos.

La técnica de recolección de datos que se implementó fue la entrevista a profundidad porque permite entender las significaciones simbólicas de la otredad, mediante un intercambio emocional durante el encuentro cara a cara (Schwartz \& Jacobs, 1995). Pero esto no significa que se hable de cualquier tema sin obedecer una estructura. Se maneja una Guía Temática que permite llevar a cabo la entrevista de una manera más holgada, pero sin perder de vista aquellos temas que interesan (Delgado y Gutiérrez, 1998). La Guía de Entrevista se ejecuta de manera flexible, permitiendo que el entrevistado se explaye sin que se aleje de los temas de investigación. 
El número de investigadores entrevistados dependió de la Saturación Teórica, la cual se alcanza cuando los datos comienzan a ser repetitivos, las categorías de análisis se saturan y se llega a la comprensión del fenómeno estudiado (Strauss y Corbin, 2002). Para llegar a la saturación de datos, se empleó un principio que es aritmético, porque lo que se pretende con la saturación es alcanzar la media. Este principio tuvo sentido una vez que los datos arrojados por las entrevistas comenzaron a ser iterativos. En ese momento, se detuvieron las entrevistas porque los informantes ya no tenían nada novedoso que aportar a la investigación.

Para reclutar a los informantes se utilizó la técnica de bola de nieve. Primero se contactó a un tratante de extraños para que nos introdujera en el escenario de estudio y nos pusiera en contacto con los primeros informantes. Después esos informantes nos ayudaron a conocer a otros informantes, logrando así un efecto de bola de nieve (Taylor y Bogdan, 1987). Se contactaron fueron profesores-investigadores que actualmente están participando en procesos de transferencia de conocimiento con la capacidad oral de transmitir la información, la disposición para colaborar y el tiempo para corroborar los datos (Rodríguez et al., 1996).

\section{Presentación y discusión de los resultados}

El clima organizacional está conformado por normas, valores y creencias que influyen en el comportamiento de los integrantes de una organización y en los procesos de interacción social que se dan en su interior (García, 2018). Esto quiere decir que aspectos de la estructura burocrática y la cultura organizacional de las Universidades Públicas Estatales determinan el proceder de los individuos que las conforman. De acuerdo con E3, los marcos legales de la universidad de adscripción influyen en las decisiones que los miembros de la organización toman respecto a la transferencia tecnológica. Desde su perspectiva, las normas actuales impiden que la rectoría tome la decisión de crear una Oficina de Transferencia Tecnológica:

La universidad tiene en mente abrir una Oficina de Transferencia Tecnológica, pero hasta la fecha no ha podido concretar ese proyecto. Sabe que es muy importante, eso sí. De hecho, ha sido sede de varios congresos a nivel internacional para el tema de la transferencia de tecnología, pero no ha tenido todavía los recursos y tal vez el tiempo para poder, entre todas las funciones que está cubriendo, abrir una oficina de ese tipo. [...] una de las críticas más fuertes que nos hacen cuando hablamos de transferencia de tecnología es la falta de producción de patentes. En ciencia hay publicaciones en papers y hay patentes. Pero la métrica de patentes siempre ha sido muy baja en nuestra universidad y en sí en México. Entonces, cuando el IMPI habla de estos temas siempre nos pone como los 
villanos más nefatos de la historia de la ciencia en el país porque considera que las universidades públicas están rezagadas en este tema. Ellos argumentan que no patentamos ni transferimos conocimiento y que por lo tanto no servimos a la sociedad. Pero hay que entender que una patente no es un proceso que se haga en un mes. Es un proceso que se lleva años para poder generarse. Entonces, la universidad estuvo en algún momento de poner su propia Oficina de Transferencia Tecnológica para propulsar el asunto de las patentes, pero es algo que todavía en el marco legal y político de la universidad lleva su tiempo y su cabildeo [...]. La cantidad de recursos humanos que la universidad tiene asignados para este asunto de las cuestiones legales está muy saturada y eso hace que los procesos sean más lentos (E3, comunicación personal,20/02/15).

Para el entrevistado E3, uno de los problemas que impide que el marco legal de la universidad cambie para respaldar la transferencia tecnológica es la falta de personal jurídico suficiente para trabajar en la reforma. De acuerdo con E3, este retraso les ha acarreado críticas del exterior porque desde la perspectiva de instituciones como el IMPI, las universidades públicas del país están atrasadas en el asunto del patentamiento y la transferencia de conocimiento por una falta de voluntad de las autoridades universitarias. Esta situación obstaculiza su labor como productores de conocimiento transferible porque tienen que buscar de manera solitaria estrategias para lograr sus metas.

Para Montoya et al. (2017), el clima organizacional se define como la percepción compartida por los miembros de una organización del ambiente de trabajo; es la representación o la interpretación intersubjetiva que tienen de una organización sus integrantes y del impacto que los elementos objetivos organizacionales tienen en su toma de decisiones y expectativas profesionales. Esto significa que los miembros de una organización actúan a partir de lo que creen, en base a sus experiencias cotidianas, de la organización donde laboran. El profesor-investigador E1 expresa la percepción que tiene de su universidad, la cual revela una actitud crítica hacia la organización por el poco apoyo que le ha proporcionado para lograr la transferencia de sus tecnologías:

[...] Aquí la Oficina de Transferencia Tecnológica no me ha apoyado en nada, menos de cero. Les he pedido ayuda para cosas básicas, ¿me entiendes? Y no me lo dan. Es una política equivocada. En realidad, si tú puedes conseguir el $70 \%$ del dinero y la universidad aporta el 30\%, pues la universidad aporta 30 pesos y gana 100. Tú tienes un millón de pesos y la universidad pone 300 mil y aumenta la propia riqueza de la universidad a un millón de pesos, en salarios y en todo. Pero no lo entienden; no comprenden el potencial de vender el conocimiento. Si tú les pides el dinero aquí se mueren de la risa [...].Si quieres mover algo y cambiar los esquemas institucionales con cosas como las que 
estoy haciendo yo, tienes que entender que vas a afectar intereses de personas conservadoras que lo único que hacen todo el día es tomar café. Y publicar un articulillo por ahí y repetir cosas intrascendentes que ya se han dicho otros en la ciencia. ¡Qué estúpido!, ¿no crees?... (E1, comunicación personal,29/09/14).

En el entrevistado E1 se percibe una actitud de inconformidad ante lo que desde su perspectiva es incompetencia, rigidez y falta de seriedad de su universidad respecto a la transferencia de conocimiento. Desde su percepción, no comprende el potencial de la transferencia tecnológica porque está enquistada en prácticas que están en el marco del Modo I de producción de conocimiento (Gibbons et al., 1997), el cual privilegia la generación de conocimiento acumulativo y no la innovación. La percepción de E1 de su universidad afecta su actitud ante sus prácticas, las cuales desde su perspectiva obstaculizan su labor como investigador pionero en su disciplina. Ante un clima organizacional adverso, el entrevistado E1 tiene que buscar alternativas de financiamiento para su proyecto de innovación.

Bordas (2016) señala que el clima organizacional puede o no satisfacer las expectativas de los miembros de una organización y favorecer o no el reconocimiento de su desempeño laboral. Si el trabajador se encuentra satisfecho con la organización, su comportamiento será conveniente para el cumplimiento de las metas organizacionales, pero si el empleado percibe que su esfuerzo no es valorado, entonces, existe la posibilidad de que surjan conflictos o prevalezca un estado de desánimo y apatía. De acuerdo con E2, su labor como científica es poco valorada por su universidad porque en el país se privilegia el financiamiento para ciencia básica y no para ciencia aplicada. Esto la lleva a costear sus propios proyectos tecnológicos:

[...] el diseño de una antena requiere de la aplicación de geometría básica y electrónica avanzada, pero que aunado a la integración inteligente de material de buena calidad generamos algo que es rentable y económico, aunque gastemos en material sofisticado. Entonces, son tareas que parecen muy sencillas, pero que aún dentro de esa sencillez su implementación requiere pericia, habilidad y sobretodo dinero. Hay que comprar materiales avanzados. Hay que incluso buscar softwares adecuados. Y pues bueno, cabe mencionar que mucho del financiamiento corre por nuestra cuenta. Cuando nosotros decimos "ay, quiero hacer algo práctico. Voy a hacer algo nuevo como una antena" no es algo que se me financia con recursos públicos, como se hace con la ciencia básica. Algunos lo ven como si no tuviera ciencia detrás, ¿no? Y por lo tanto no es apoyado por ningún organismo tradicional. Eso hace que tomemos la decisión de autofinanciarnos [...]. De hecho, he adquirido mucho del bolsillo propio: materiales y diversas cosas, para poder salir adelante (E2, comunicación personal,11/02/15). 
Segredo (2013) sostiene que el clima organizacional de una institución como la Universidad Pública Estatal se construye a partir de las percepciones de los sujetos, las cuales están influidas por factores internos y externos a la organización. El entrevistado E8 relata que en su universidad existen estructuras que están llevando a cabo actividades de transferencia de conocimiento. Pero su labor se ve entorpecida por la percepción que tienen algunos empresarios de su quehacer científico, por considerar que el objetivo de la producción científica universitaria es la divulgación pública de los resultados de investigación y no la protección de la propiedad intelectual. Esto desde su perspectiva desfavorece su propósito lucrativo, que es aplicar conocimiento insólito que esté protegido contra toda divulgación en la sociedad, ya que si se da a conocer pierden ventajas competitivas:

[...] creo que la falta de vinculación con las empresas de alta tecnología es una de las grandes debilidades que tenemos como universidad pública [...] Tenemos aquí dos centros de vinculación con la industria. Uno es el ID [...] que dirige el doctor $\mathrm{A}[. .$.$] . Es un instituto que tiene la finalidad de desarrollar tecnología$ innovadora para la pequeña y mediana empresa. Y otro se llama C [...] Hasta donde tengo entendido es un centro de investigación e innovación de actividades empresariales. Ese todavía no está tan consolidado como el otro. El ID [...] trabaja en función de lo que hacen todas las universidades públicas, que es lograr la vinculación con empresarios a través de la incubación de empresas. Se hacen también diagnósticos de las empresas y eventualmente se logra un plan de implementación para la mejora competitiva de la empresa. Nada más que un punto quizá de debilidad es que la industria de tecnología media y alta no se arriesga porque dicen "bueno, yo tengo al investigador $\mathrm{X}$ o $\mathrm{Y}$ del $\mathrm{C}[\ldots]$ o del $\mathrm{C}[\ldots]$, pero es un investigador que trabaja en la divulgación del conocimiento. Y se guardan su intención de vincularse con la universidad por temor a perder exclusividad del conocimiento producido (E8, comunicación personal, 19/02/15).

La actitud de este tipo de empresarios respecto a la labor de los investigadores de las universidades públicas afecta el clima organizacional de la institución y por ende su desempeño como factor de la innovación. El entrevistado E8 percibe que las instancias que existen dentro de su universidad para la transferencia de conocimiento aún son incipientes en cuanto a la vinculación con las empresas porque considera que les falta construir relaciones con estas organizaciones. En este contexto, es probable que haga falta la creación de una Oficina de Transferencia Tecnológica con personal capacitado en la gestión del conocimiento para ayudar a mejorar las relaciones con la industria. E8 reconoce que en su universidad se están haciendo esfuerzos para lograr la vinculación, pero también sabe que faltan cosas por hacer. 
Brunner (2011) señala que las universidades que tienen la capacidad de transformar su forma de gestión administrativa y su estructura burocrática para adaptarse a su medio ambiente son organizaciones que tienen una gobernanza que responde a las necesidades de la sociedad post-industrial. Las universidades que desean ser emprendedoras necesitan ejercer un tipo de gobernanza en el que sus estructuras cambien y se adapten a las nuevas circunstancias de la sociedad del riesgo (Beck, 1997). Una sociedad en la que las amenazas provocadas por las actividades humanas necesitan de estrategias sustentables para evitar sus efectos globales. Para el investigador E8, una universidad con una gobernanza emprendedora facilita la vinculación, la generación de conocimiento sustentable y la innovación.

De acuerdo con el profesor-investigador E9, la universidad de adscripción lo está apoyando en su proyecto de investigación; incluso, desde su perspectiva existe "gente con visión" que en su institución lo están motivando a continuar con su labor. Pero la burocracia universitaria es en su opinión un obstáculo para su quehacer científico porque los trámites que tiene que realizar para gestionar recursos son ineficientes, engorros y lentos. En el discurso del entrevistado se percibe un sentimiento de lealtad y agradecimiento hacia su universidad. Aunque al mismo tiempo tenga que reconocer que el obstáculo que impide el buen término de sus proyectos es la burocracia y la consecuente falta de estructuras flexibles que promuevan el emprendimiento:

Si le tengo mucho que agradecer a la Universidad de Guadalajara. Hay gente en la institución que si tiene visión. Si hay gente que es impulsora de la ciencia. Aunque bueno, los trámites siempre son engorrosos y siempre quitan tiempo. Ese sería un inconveniente. Se tienen que eficientar. Yo creo que esta es una problemática nacional e histórica que la traemos arrastrando desde hace mucho, pero tenemos que empezar a cambiar. Uno pierde mucho tiempo en burocracias. Uno pierde mucho tiempo en procesos burocráticos ineficientes. Definitivamente, los procesos burocráticos en la universidad tienen que modernizarse y ser más eficientes para el beneficio de todos. Pero a pesar de que son complicados no han sido pretexto para decir no voy, no trabajo o no lo hago [...]. Simplemente ya sé que son engorrosos y que tengo que planear mis tiempos y tener paciencia. Eso sí, me puede costar dos mañanas perdidas que puedo trabajar en un artículo sobre el proyecto [...]. Yo siento que es una cuestión de eficiencia [...] (E9, comunicación personal, (26/02/15).

Si se quiere emprender un proyecto de reforma universitaria para mejorar los modelos de gestión y gobierno, con la finalidad de modernizar a la universidad y convertirla en una organización con responsabilidad social, se necesita saber si la organización está destinando recursos en áreas de importancia estratégica y si lo está haciendo 
de manera eficiente (Santiago, Trembaly, Basri y Arnal, 2008). De acuerdo con E9, a su universidad de adscripción le hace falta invertir recursos para mejorar su modelo de gobierno porque los procesos burocráticos son rígidos y generan malestar en los investigadores que desean agilizar sus proyectos de desarrollo tecnológico. En la siguiente figura, se muestra la manera en que el clima organizacional afecta en la construcción de las trayectorias profesionales de los investigadores entrevistados.

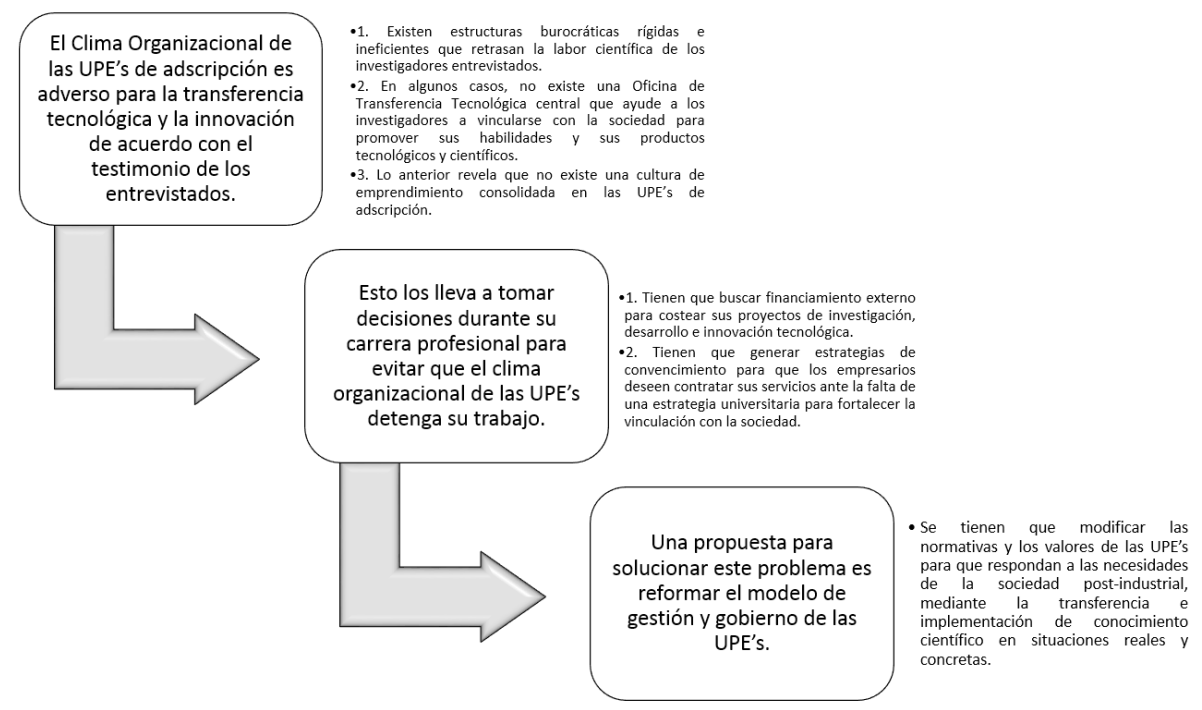

Figura 1. El Clima Organizacional de las UPE's y su efecto en la construcción de las trayectorias académico-profesionales de los investigadores emprendedores. Elaboración propia a partir de los testimonios de los profesores-investigadores entrevistados.

\section{Reflexiones finales}

Las trayectorias académico-profesionales de los profesores-investigadores entrevistados son construidas a partir de la influencia que ejercen en su comportamiento los factores objetivos de las Universidades Públicas Estatales de adscripción, los cuales tienen un rol obstaculizador de la transferencia de conocimiento, la vinculación y la innovación por estar ausentes o ser ineficientes y rígidas. De acuerdo con los testimonios de los sujetos de estudio, la estructura burocrática de las universidades de adscripción no responde de manera adecuada a sus expectativas como científicos que participan en procesos de transferencia de conocimiento porque sus rutinas y normativas son rígidas, lo que impide que se dé de manera fluida la vinculación con la sociedad, mediante la producción de conocimiento innovador. 
Desde su perspectiva, la burocracia universitaria es lenta y poco eficaz. Esto provoca que la universidad pública en nuestro país aún esté lejos de asimilar el modelo de la universidad emprendedora que existe en países como EEUU. Las universidades de adscripción permiten que los investigadores entrevistados lleven a cabo actividades de transferencia tecnológica, pero no les otorgan los apoyos suficientes para cumplir sus metas como científicos pioneros en su campo. De acuerdo con sus testimonios, existe el apoyo de las autoridades universitarias a su labor como investigadores innovadores, pero es un apoyo superficial porque no existen las estructuras adecuadas para las labores de producción científica y transferencia de conocimiento ni una cultura de emprendimiento que fomente programas de estímulos para la innovación. Si existen estas estructuras, son incipientes o están gestionadas por personal poco capacitado.

Ante el clima organizacional desfavorable de las universidades de adscripción, los investigadores entrevistados dirigen su trayectoria profesional hacia un puerto seguro, buscando sus propios recursos para financiar sus proyectos de investigación. Esto implica riesgos para ellos en lo profesional. Aun así ellos deciden asumirlos y enfrentar las dificultades de un sistema burocrático universitario lento, rígido, anacrónico y vertical. De igual manera, los investigadores entrevistados generan sus propias estrategias de construcción de redes de colaboración científica, las cuales incluyen tácticas de convencimiento para sumar a empresarios, a pesar de los prejuicios respecto al papel del investigador universitario.

\section{Referencias:}

Beck, U. (1997). La teoría de la sociedad del riesgo reformulada. Revista Polis: Investigación y Análisis Sociopolítico y Psicosocial, (97), 171-196. Disponível em http://tesiuami.uam.mx/revistasuam/polis/ viewissue.php?id=15, consultado em Março de 2018.

Bermúdez-Aponte, J. J., Pedraza, A., y Rincón, C. I. (2015). El clima organizacional en universidades de Bogotá desde la perspectiva de los estudiantes. Revista Electrónica de Investigación Educativa, 17(3), 1-12. Disponível em http://redie.uabc.mx/vol17no3/contenido-bermudezetal.html, consultado em Março de 2018.

Bordas, M. J. (2016). Gestión Estratégica del Clima Laboral. Madrid, España: Uned.

Brunner, J. J. (2011). Gobernanza universitaria: tipología, dinámicas y tendencias. Revista de Educación, (355), 137-159. Disponível em http://www.revistaeducacion.educacion.es/re355/re355_06.pdf , consultado em Março de 2018.

Clark, B. R. (1998). Creating Entrepreneurial Universities. EEUU: Oxford, Pergamon Press.

Cortés, F. (2000). Algunos aspectos de la controversia entre investigación cualitativa e investigación cuantitativa. Argumentos, (36), 81-108. Disponível em http://148.206.107.15/ biblioteca_digital/estadistica. php?id_host=6\&tipo=ARTICULO\&id=2890\&archivo $=1$ - 178 2890 pcg.pdf\&titulo=Algunos $\% 20$ aspectos $\% 20$ de $\% 20 l a \% 20$ controversia $\% 20$ entre $\% 20$ investigaci\%C3\%B3n\%20cualitativa\%20e\%20investigaci\%C3\%B3n\%20cuantitativa, consultado em Março de 2018. 
De Arteche, M., Santucci, M., y Welsh, S.V. (2013). Redes y clusters para la innovación y la transferencia del conocimiento. Impacto en el crecimiento regional en Argentina. Estudios Gerenciales. Elsevier España, (29), 127-138. Disponível em http://www.elsevier.es/es-revista-estudios-gerenciales-354articulo-redes-iclusters-i-innovacion-transferencia-del-90231795, consultado em Março de 2018.

Delgado, J. M., y Gutiérrez J. (Ed.). (1998). Métodos y Técnicas Cualitativas de Investigación en Ciencias Sociales. Madrid, España: Editorial Síntesis. Disponível em https://dialnet.unirioja.es/servlet/ libro?codigo=491811, consultado em Março de 2018.

Denzin, N.K., y Lincoln, Y.S. (2000). The SAGE Handbook of Qualitative Research. London: Sage Publications.

Etzkowitz, H. (2003). Research groups as 'quasi-firms': The invention of the enterpreneurial university. Research Policy, 32(1), 109-121. Doi: https://doi.org/10.1016/S0048-7333(02)00009-4.

Galindo, J., Sanz, P., y De Benito, J. J. (2011). La universidad ante el reto de la transferencia del conocimiento 2.0: análisis de las herramientas digitales a disposición del gestor de transferencia. Investigaciones Europeas de Dirección y Economía de la Empresa, 17(3), 111-126. Doi: https://doi. org/10.1016/S1135-2523(12)60123-3.

García, E. (2018). Clima organizacional en estaciones de servicio de la Goajira colombiana. Revista de publicaciones científicas y académicas, 2(2), 42-61. Disponível em file:///C:/Users/Alumno34/ Downloads/21-Article\%20Text-86-1-10-20190220\%20(1).pdf, consultado em Março de 2018.

Gibbons, M., Limoges, C., Nowotny, H., Schwartzman, S., Scott, P., y Trow, M. (1997). La nueva producción del conocimiento. La dinámica de la ciencia y la investigación en las sociedades contemporáneas. Barcelona: Ediciones Pomares-Corredor.

Guerrero, M., y Urbano, D. (2012). Transferencia de conocimiento y tecnología. Mejores prácticas en las universidades emprendedoras españolas. Revista Gestión y Política Pública, 21(01), 107-139. Disponível em http://www.scielo.org.mx/pdf/gpp/v21n1/v21n1a4.pdf , consultado em Março de 2018.

Hernández, R., Fernández, C., y Baptista, P. (2006). Metodología de la investigación. DF, México: Mc Graw Hill Interamericana.

Jiménez, M. S. (2009). Tendencias y hallazgos en los estudios de trayectoria: una opción metodológica para clasificar el desarrollo laboral. Revista Electrónica de Investigación Educativa, 11(1), 1-21. Disponível em http://redie.uabc.mx/vol11no1/contenido-jimenez.html , consultado em Março de 2018.

Lee, J., \& Win, H. N. (2004). Technology Transfer between University Research Centers and Industry in Singapore. Technovation, 24(5), 433-442. Doi: https://doi.org/10.1016/S0166-4972(02)00101-3.

Montoya, P., Bello, N., Bermúdez, N., Burgos, F., Fuentealba, M., y Padilla, A. (2017). Satisfacción Laboral y su Relación con el Clima Organizacional en Funcionarios de una Universidad Estatal Chilena. Revista Ciencia y Trabajo, 19(58), 7-13. Disponível em https://scielo.conicyt.cl/pdf/cyt/ v19n58/0718-2449-cyt-19-58-00007.pdf, consultado em Março de 2018.

Rizo, M. (2006). La interacción y la comunicación desde los enfoques de la psicología social y la sociología fenomenológica. Breve exploración teórica. Anàlisi: Quaderns de comunicació i cultura, (33), 45-62. Disponível em http://ddd.uab.cat/pub/analisi/02112175n33/02112175n33p45.pdf, consultado em Março de 2018. 
Rodríguez, G., Gil, J., y García, G. (1996). Metodología de la Investigación Cualitativa. Málaga: Ediciones Aljibe.

Santiago, P., Trembaly, K., Basri, E., \& Arnal, E. (2008). Tertiary Education for the Knowledge Society (Vol. 2), Paris: Organisation for Economic Development and Co-Operation.

Schwartz, H., y Jacobs, J. (1995). Sociología Cualitativa, Método para la reconstrucción de la realidad. DF, México: Editorial Trillas.

Segredo, A. M. (2013). Clima organizacional en la gestión del cambio para el desarrollo de la organización. Revista Cubana de Salud Pública, 39(2), 385-393. Disponível em http://www.redalyc.org/articulo. oa?id=21430556017, consultado em Março de 2017.

Strauss, A., y Corbin, J. (2002). Bases de la investigación cualitativa. Antioquía, Colombia: Facultad de Enfermería de la Universidad de Antioquía.

Taylor, S.J., y Bogdan, R. (1987). Introducción a los Métodos Cualitativos de Investigación. Barcelona: Paidós Básica.

Armando Miranda Zea

Doctor en Ciencias Sociales por la Universidad Autónoma del Estado de Morelos Catedrático de Periodismo y Comunicación Del Centro Universitario Tlacaélel (CUT) Email: hiperion82@hotmail.com

Omar García Ponce de León

Doctor en Sociología por la Universidad de Barcelona. Profesor-investigador del Instituto de Ciencias de la Educación (ICE) de la Universidad Autónoma del Estado de Morelos.

Email: omartqu@yahoo.com

Ricardo Pérez Mora

Doctor en Educación por el Centro Universitario de Ciencias Sociales y Humanidades de la Universidad de Guadalajara. Jefe de Departamento de Políticas Públicas perteneciente al Centro Universitario de Ciencias Económico Administrativas Email: ricardo.perez@cucea.udg.mx

Correspondência

Ricardo Pérez Mora

Departamento de Políticas Públicas

Centro Universitario de Ciencias Económico Administrativas Periférico Norte № 799, Núcleo Universitario Los Belenes 45100 - Zapopan, Jalisco (México) 